\title{
Trimeron-phonon coupling in magnetite
}

\author{
Przemysław Piekarz, ${ }^{1}$ Dominik Legut, ${ }^{2}$ Edoardo Baldini ${ }^{3}$ Carina A. Belvin,${ }^{3}$ Tomasz \\ Kołodziej, ${ }^{4}$ Wojciech Tabiś, ${ }^{5}$ Andrzej Kozłowski,${ }^{5}$ Zbigniew Kakol, ${ }^{5}$ Zbigniew Tarnawski, ${ }^{5}$ José \\ Lorenzana, ${ }^{6}$ Nuh Gedik, ${ }^{3}$ Andrzej M. Oleś, ${ }^{7,8}$ Jürgen M. Honig, ${ }^{9}$ and Krzysztof Parlinski ${ }^{1}$ \\ ${ }^{1}$ Institute of Nuclear Physics, Polish Academy of Sciences, Radzikowskiego 152, 31-342 Kraków, Poland \\ ${ }^{2}$ IT4Innovations and Nanotechnology Center, VSB-Technical University of Ostrava, \\ 17.listopadu 2172/15, 70800 Ostrava-Poruba, Czech Republic \\ ${ }^{3}$ Department of Physics, Massachusetts Institute of Technology, Cambridge, Massachusetts 02139, USA \\ ${ }^{4}$ SOLARIS National Synchrotron Radiation Centre, Czerwone Maki 98, 30-392 Kraków, Poland \\ ${ }^{5}$ AGH University of Science and Technology, Faculty of Physics and Applied Computer Science, 30-059 Kraków, Poland \\ ${ }^{6}$ La Sapienza University, 00-185 Rome, Italy \\ ${ }^{7}$ Institute of Theoretical Physics, Jagiellonian University, Profesora Stanistawa Eojasiewicza 11, 30-348 Kraków, Poland \\ ${ }^{8}$ Max Planck Institute for Solid State Research, Heisenbergstrasse 1, 70569 Stuttgart, Germany \\ ${ }^{9}$ Department of Chemistry, Purdue University, West Lafayette, Indiana 47907-2084, USA
}

(Dated: October 23, 2020)

\begin{abstract}
Using density functional theory, we study the lattice dynamical properties of magnetite $\left(\mathrm{Fe}_{3} \mathrm{O}_{4}\right)$ in the high-temperature cubic and low-temperature monoclinic phases. The calculated phonon dispersion curves and phonon density of states are compared with the available experimental data obtained by inelastic neutron, inelastic x-ray, and nuclear inelastic scattering. We find a very good agreement between the theoretical and experimental results for the monoclinic $C c$ structure revealing the strong coupling between charge-orbital (trimeron) order and specific phonon modes. For the cubic phase, clear discrepancies arise which, remarkably, can be understood assuming that the strong trimeron-phonon coupling can be extended above the Verwey transition, with lattice dynamics influenced by the short-range trimeron order instead of the average cubic structure. Our results establish the validity of trimerons (and trimeron-phonon coupling) in explaining the physics of magnetite much beyond their original formulation.
\end{abstract}

\section{INTRODUCTION}

The Verwey transition in magnetite - in which the electrical conductivity changes discontinuously by two orders of magnitude $[1,2]$ - is one of the most intriguing and extensively studied phenomena in condensed matter physics. The exceptional character of the phase transition stems from a cooperative mechanism involving charge, orbital, spin, and lattice degrees of freedom. Above the transition at $T_{\mathrm{V}}=124 \mathrm{~K}$, magnetite crystallizes in the cubic $F d \overline{3} m$ structure [3] with the tetrahedrally coordinated $A$-sites occupied by $\mathrm{Fe}^{3+}$ ions and octahedrally coordinated $B$-sites with the mixed valency $\mathrm{Fe}^{2.5+}$. At $T_{\mathrm{V}}$, magnetite exhibits a structural phase transition [4-9] from the cubic $F d \overline{3} m$ to the monoclinic Cc symmetry (see Fig. 1) [10].

To explain this transition, Verwey postulated a purely electronic mechanism of charge ordering, where the (001) planes are alternatively occupied by $\mathrm{Fe}_{B}^{2+}$ and $\mathrm{Fe}_{B}^{3+}$ ions below $T_{\mathrm{V}}$. Subsequently, this simple model was questioned on the basis of diffraction studies [4-7] and a more complex charge-orbital (CO) order with 16 nonequivalent $B$ sites was identified below $T_{\mathrm{V}}$ [8-19]. As demonstrated by density functional theory (DFT) studies, the CO order is a consequence of strong local electron interactions within the $3 d$ states and the coupling of electrons to lattice distortions [20-26]. This electron-lattice cooperative behavior results in a complicated pattern composed of three-site polarons, called trimerons [10, 27]. In each trimeron two end $\mathrm{Fe}^{3+}$ cations are shifted towards a cen- tral $\mathrm{Fe}^{2+}$ cation [28]. Recently, the soft electronic fluctuations of the trimeron order showing critical behavior close to the Verwey transition have been discovered [29]. These fluctuations were described through a polaron tunneling model [30], which involves the coupling between the trimeron structure and phonons.

The interaction between electronic and lattice degrees

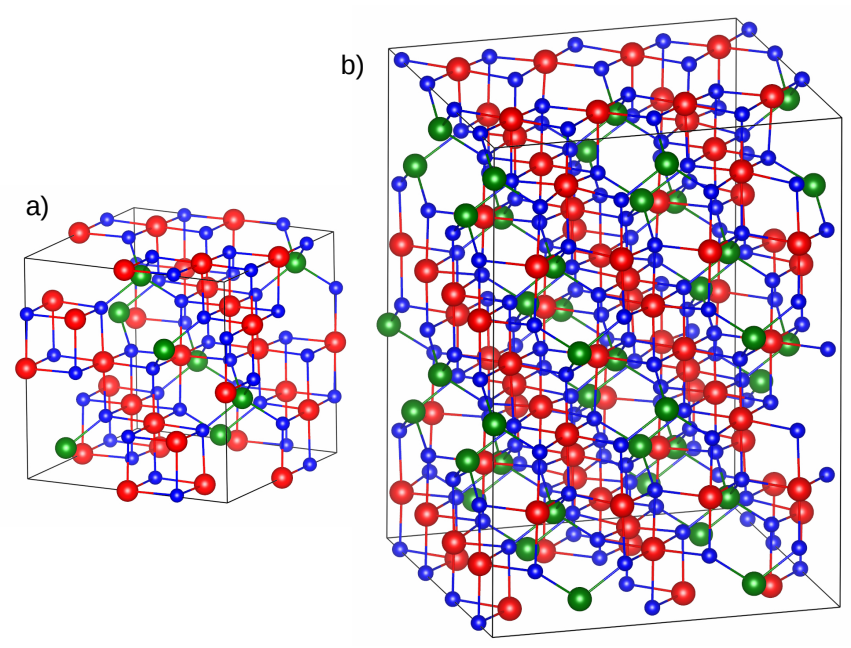

FIG. 1. The unit cells of magnetite in: (a) cubic $F d \overline{3} m$ and (b) monoclinic $C c$ symmetry. Green, red, and blue spheres represent $\mathrm{Fe}_{A}, \mathrm{Fe}_{B}$, and $\mathrm{O}$ atoms, respectively. 
of freedom is reflected in the precursor effects observed above $T_{\mathrm{V}}$. The critical behavior of the elastic constant $c_{44}$, which softens from room temperature to $T_{\mathrm{V}}[31,32]$, was explained in terms of bilinear coupling of the elastic strain to a fluctuation mode of the charge ordering field of $T_{2 g}$ symmetry [32]. Critical fluctuations above $T_{\mathrm{V}}$ were investigated by diffuse scattering of electrons [33], neutrons [34-37], and x-rays [38]. Neutron measurements revealed the diffuse scattering at commensurate points in reciprocal space, i.e., at the same wave vectors as the superlattice reflections of the monoclinic phase, only a few kelvins above $T_{\mathrm{V}}$ [34].

However, even more intense maxima and in a much broader range of temperatures (even at room temperature) were found at incommensurate $\boldsymbol{q}$ vectors close to the $\Gamma$ and $X$ points of the Brillouin zone [35-37]. Shapiro et al. [35] showed that neutron diffuse scattering centered at zero energy is coupled with transverse acoustic (TA) phonons. This electron-phonon coupling induces anomalous anharmonic behavior of the TA modes, with the largest phonon broadening found at incommensurate $\boldsymbol{q}$ points [39].

It is remarkable that an extended x-ray absorption fine structure (EXAFS) study [40] provided direct evidence that local distortions related to atomic displacements of the monoclinic phase are robust and remain unaltered during the transition. Recently, an x-ray pair distribution function analysis has revealed that local structural fluctuations persist up to the Curie transition at $T_{C}=850 \mathrm{~K}$ [41]. This short-range order (SRO), first postulated by Anderson [42], was also identified via several probes: electric conductivity [43], muon-spinrelaxation [44], optical conductivity [45], photoemission [46], magneto-optical [47], and resonant inelastic x-ray scattering (RIXS) studies [48].

All the results mentioned above indicate the important role of the electron-phonon coupling which modifies the transport properties of magnetite, and may initiate the structural transition from the cubic to the monoclinic phase. In the theory this coupling was introduced by Yamada who proposed that the mechanism behind the Verwey transition involves the condensation of charge order fluctuations coupled to a TA phonon mode with $\Delta_{5}$ symmetry [49]. Indeed, studies based on DFT calculations demonstrated that phonons with $\Delta_{5}, X_{3}$, and $X_{4}$ symmetries strongly couple to the electronic states and may induce the monoclinic deformation [23, 24, 39]. According to a detailed refinement, the low-temperature structure of magnetite can be described by a subset of phonon modes at the $\Gamma, \Delta, X$, and $W$ points of the Brillouin zone $[16,50,51]$.

Experimentally, the phonon dispersion curves in magnetite were studied by inelastic neutron scattering (INS) [52-54] and by inelastic x-ray scattering (IXS) [39]. The Fe-projected phonon density of states (DOS) was measured using nuclear inelastic scattering (NIS) [55-57] and detailed studies of the optical modes at the Brillouin zone center were performed by spontaneous Raman scatter- ing and infrared reflectivity measurements [58-67]. The presence of phonon anharmonicity and strong electronphonon coupling was also demonstrated by means of Raman measurements [65]. Anomalies of the lattice vibrations that originate from strong coupling to electronic excitations and the spectroscopic signatures of diffusive modes in the electronic contribution to the Raman response function were observed [66]. Ultrafast broadband optical spectroscopy revealed that impulsive photoexcitation of particle-hole pairs couples to the fluctuations of the ordering field and coherently generates phonon modes of the ordered phase above $T_{\mathrm{V}}$ [67]. The existence of long-lived precursor fluctuations was further evidenced by recent INS studies [68].

Since the lattice dynamics is strongly coupled to the electronic system and the SRO in the high-temperature phase of magnetite, it is important to explore how the phonon spectrum changes at the stuctural phase transition and how well these changes can be described within the DFT framework. To this end, in this paper, we analyze the phonon spectra calculated for the $F d \overline{3} m$ cubic and the $C c$ monoclinic structures. The results obtained for the monoclinic phase are in excellent agreement with the experimental data. In the cubic phase, the theoretical spectrum deviates substantially from the experimental one, which remains quite similar to the one in the broken symmetry phase, indicating significant influence of the SRO on the lattice dynamics.

The paper is organized as follows. In Sec. II, the details of the calculation method are presented. The computed phonon dispersions and phonon density of states are analyzed and compared with the experimental data in Secs. III and IV, respectively. In Sec. V, the influence of the $\mathrm{CO}$ order on the phonon modes and the mean square displacements in the monoclinic phase is studied. The discussion and summary of the results are included in Sec. VI.

\section{CALCULATION METHOD}

The electronic and crystal structures of magnetite were optimized within the DFT framework implemented in the VASP program [69, 70]. The calculations were performed using the projector augmented-wave method [71] and the generalized gradient approximation (GGA) [72]. We ensured an experimentally observed ferrimagnetic order with the opposite orientation of magnetic moments within the $\mathrm{Fe}_{A}$ and $\mathrm{Fe}_{B}$ sublattices. Strong electronic interactions in the $\mathrm{Fe}(3 d)$ states were taken into account using the GGA $+U$ method [73] with the local Coulomb interaction parameter $U=4.0 \mathrm{eV}$ and Hund's exchange $J=0.8 \mathrm{eV}$.

The cubic structure was studied in the unit cell presented in Fig. 1(a) containing 56 atoms (4 primitive cells): $8 \mathrm{Fe}_{A}, 16 \mathrm{Fe}_{B}$, and $32 \mathrm{O}$ atoms. The optimized lattice parameter $a=8.45 \AA$ is slightly overestimated compared to the experimental value $(8.39 \AA)$. The crystal struc- 


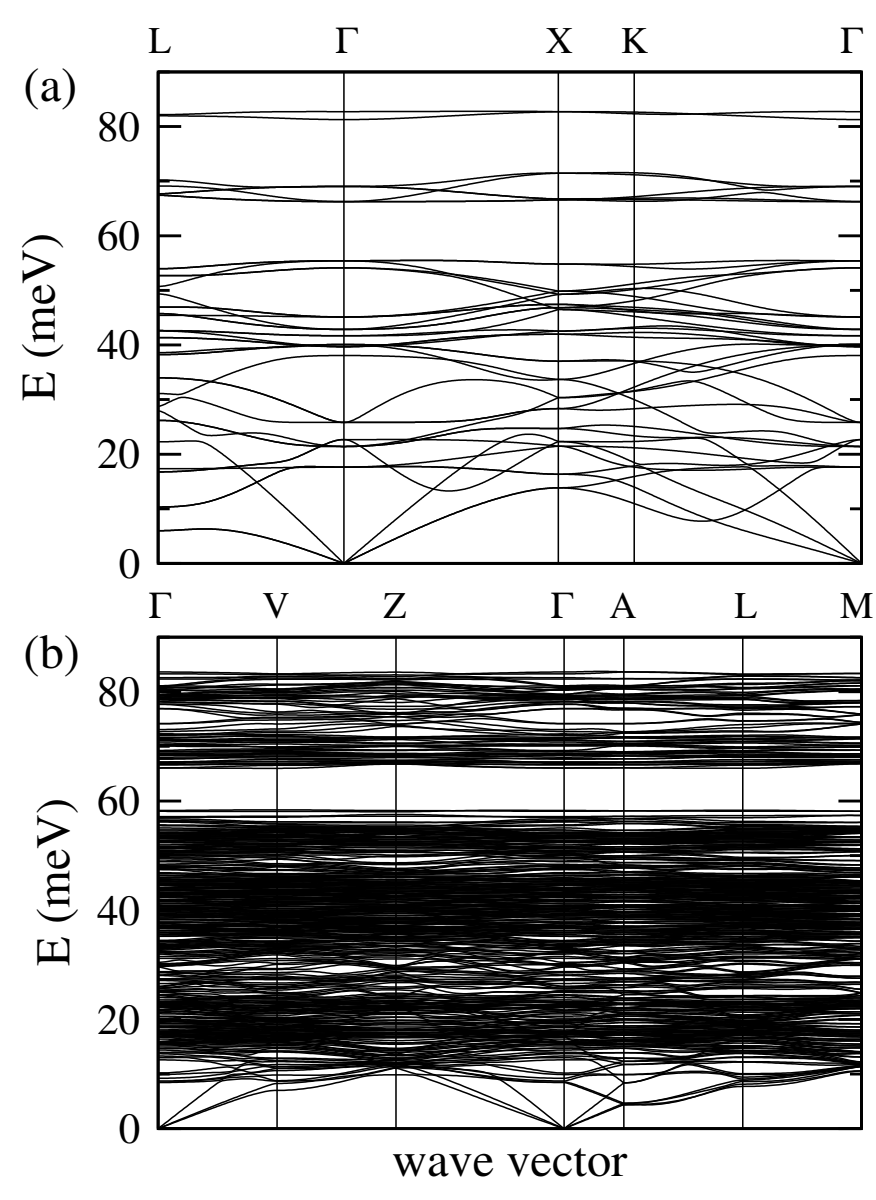

FIG. 2. The phonon dispersion curves of magnetite calculated for: (a) the cubic $F d \overline{3} m$ and (b) monoclinic $C c$ structures.

ture of the monoclinic $C c$ phase was optimized within the unit cell containing 224 atoms [see Fig. 1(b)]. The calculated lattice parameters $a=11.85 \AA, b=11.84 \AA$, $c=16.69 \AA$, and the monoclinic angle $\beta=90.20^{\circ}$ are in good agreement with the experimental data, and the obtained CO (trimeron) order agrees with the results presented in Refs. [10, 27]. Recently, we have used the same relaxed structure in the analysis of the soft charge (polaron) fluctuations of the trimeron order [29].

The phonon dispersion curves and phonon DOS were calculated by the direct method [74] using the PHONON software [75]. The Hellmann-Feynman forces were computed through the displacement of all nonequivalent atoms from their equilibrium positions and the forceconstant matrix elements were determined. By diagonalizing the dynamical matrix, the phonon dispersions along the high-symmetry directions in the first Brillouin zone were obtained. In order to evaluate properly the longitudinal optical-transverse optical (LO-TO) splitting in the monoclinic (insulating) phase, the static dielectric tensor and Born effective charges were determined using density functional perturbation theory [76].
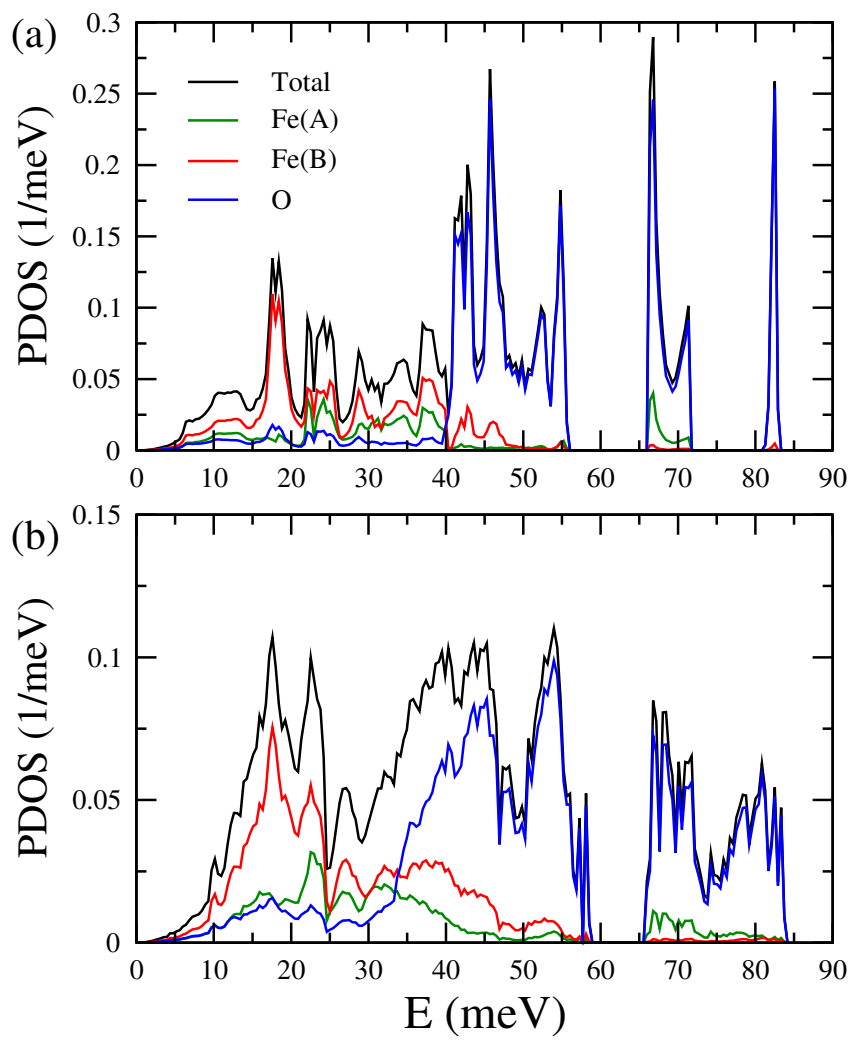

FIG. 3. The partial and total phonon DOS of magnetite calculated for: (a) the cubic $F d \overline{3} m$ and (b) monoclinic $C c$ structure.

\section{PHONON SPECTRA}

The phonon dispersion curves and phonon DOS obtained for the cubic and monoclinic phases are presented in Figs. 2 and 3. In the cubic structure, there are 42 phonon modes. All phonon energies are real (i.e., both phases are stable), although some modes show anomalous softening close to the $L$ point, along the $\Gamma-X$, and the $K-\Gamma$ directions. These anomalous dispersions induce enhanced DOS and non-Debye behavior at the lowest energies (see Fig. 3(a)). As we discuss later, this effect is related to the instability of the cubic symmetry connected with short-range distortions observed by diffuse scattering at temperatures above $T_{\mathrm{V}}$.

The low-energy range of the spectrum is dominated by vibrations of Fe atoms, with larger contribution of $\mathrm{Fe}$ atoms at the octahedral $B$ positions. The maximum of $\mathrm{Fe}$ DOS is found around $18 \mathrm{meV}$. The vibrations of $\mathrm{Fe}_{A}$ atoms dominate at higher energies for $E \in(20,40) \mathrm{meV}$. Above $40 \mathrm{meV}$, mainly $\mathrm{O}$ atoms contribute to the phonon DOS, showing an energy cut-off at $84 \mathrm{meV}$. Two energy gaps exist between $55-66 \mathrm{meV}$ and $72-81 \mathrm{meV}$, in the cubic phase. In the band located above $65 \mathrm{meV}$, there is a visible contribution from $\mathrm{Fe}_{A}$ atoms and a negligible contribution from $\mathrm{Fe}_{B}$ ones.

The primitive cell of the $C c$ structure contains 112 
atoms. Therefore, the number of phonon modes increases to 336. The partial DOS of Fe atoms is increased above $50 \mathrm{meV}$ compared to the cubic phase and they contribute to the highest modes up to $80 \mathrm{meV}$. The lower gap is reduced to about $7 \mathrm{meV}$ and the higher one is completely closed.

\section{COMPARISON WITH EXPERIMENTAL DATA}

In Fig. 4, the phonon dispersions obtained for the $C c$ structure are compared with the available experimental data. The dispersions measured above $T_{\mathrm{V}}$ by INS [53]
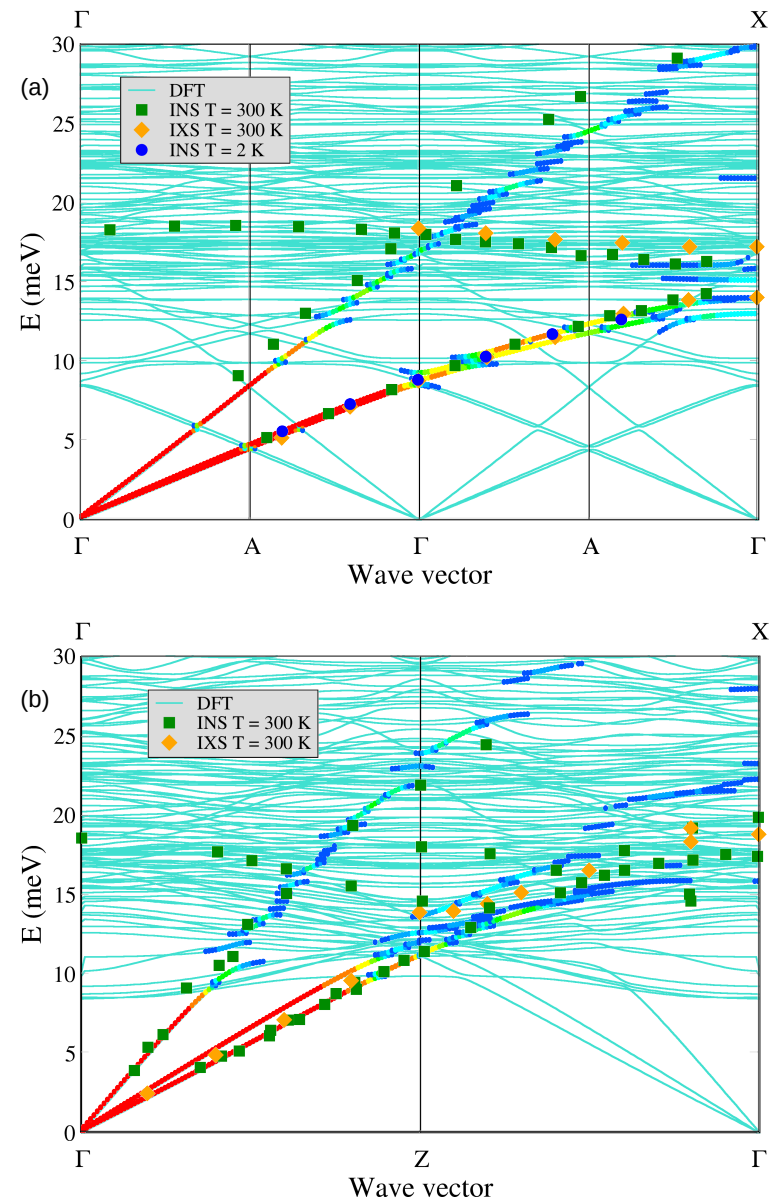

FIG. 4. The phonon dispersion curves calculated for the $C c$ structure compared with INS experimental data at $T=2 \mathrm{~K}$ taken from Refs. [53] (green squares) and [54] (blue circles) as well as IXS data [39] (orange diamonds) along: (a) the $\Delta[001]$ and (b) $\Sigma[110]$ directions of the cubic Brillouin zone. The phonon energies with the dynamical form factor above a certain threshold $t$ are plotted in blue $(t=10 \%)$, green $(t=50 \%)$, yellow $(t=70 \%)$, and red $(t=90 \%)$. The high symmetry points of the cubic (monoclinic) Brillouin zone are presented in the top (bottom) of the figures. and IXS [39] are presented in the extended Brillouin zone of the monoclinic structure. The INS measurements at $T=2 \mathrm{~K}$, well below the Verwey transition, were performed along the $\Gamma-A$ direction of the monoclinic Brillouin zone [67]. The dispersions are presented for the $\Gamma-A$ [Fig. 4(a)] and $\Gamma-Z$ [Fig. 4(b)] directions, which correspond to the $\Gamma-X \Delta[0,0,1]$ and $\Gamma-K-X \Sigma[1,1,0]$ directions in the cubic symmetry, respectively. The lowest optical modes at the $\Gamma$ point around $8 \mathrm{meV}$ result from the splitting and folding of the TA branch with $\Delta_{5}$ symmetry in the cubic structure.

Additionally, in Fig. 4, we plot the formfactor, which describes the intensity (strength) of phonon modes

$$
F(\boldsymbol{k}, j)=\left|\sum_{\alpha} \frac{e(\boldsymbol{k}, j ; \alpha)}{\sqrt{M_{\alpha}}}\right|^{2},
$$

where $\boldsymbol{e}(\boldsymbol{k}, j ; \alpha)$ is the polarization vector, $\boldsymbol{k}$ is the phonon wave vector, $j$ denotes the phonon branch, and $M_{\alpha}$ is the mass of atom $\alpha$. It can be observed that the phonon modes with the strongest intensity in the monoclinic structure correspond to the acoustic branches of the cubic structure.

A good agreement between the phonon energies measured below and above $T_{\mathrm{V}}$ with the theoretical dispersions calculated for the low-temperature $C c$ structure demonstrates that phonon energies in magnetite depend weakly on temperature. It also demonstrates that the SRO existing above $T_{\mathrm{V}}$ preserves the local geometry of the $C c$ structure. The lowest TA dispersion measured below $T_{\mathrm{V}}$ shows slightly higher values than the corresponding branch in the cubic phase [67]. We remark that the phonon dispersions calculated for the cubic phase were compared with the experimental points measured above $T_{\mathrm{V}}$ in the previous studies [23, 24, 39].

The influence of the phase transition on the partial Fe phonon DOS was studied by NIS measurements [56, 57]. The NIS spectra obtained at temperatures $T=50 \mathrm{~K}$ and $150 \mathrm{~K}$ [57] are compared in Fig. 5. The main differences are observed at the lowest energies below $10 \mathrm{meV}$

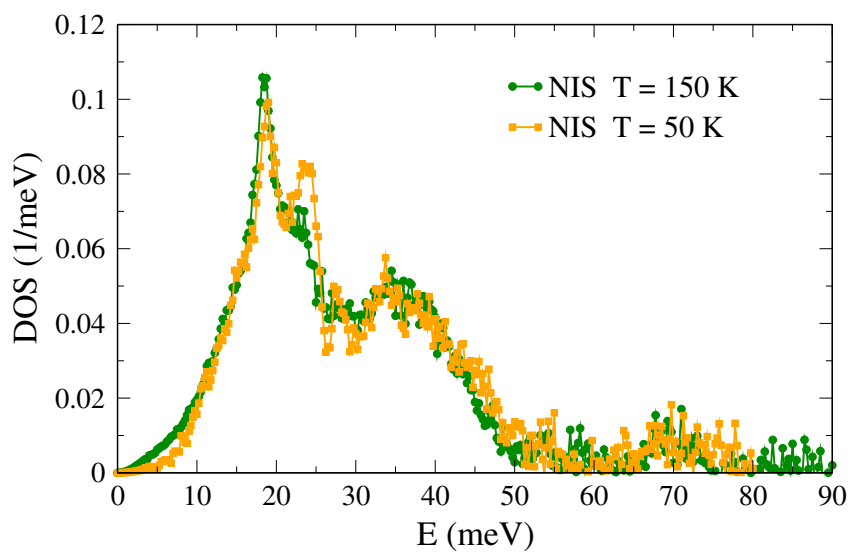

FIG. 5. The Fe-partial phonon DOS in magnetite measured by NIS at $50 \mathrm{~K}$ and $150 \mathrm{~K}$ taken from Ref. [57]. 

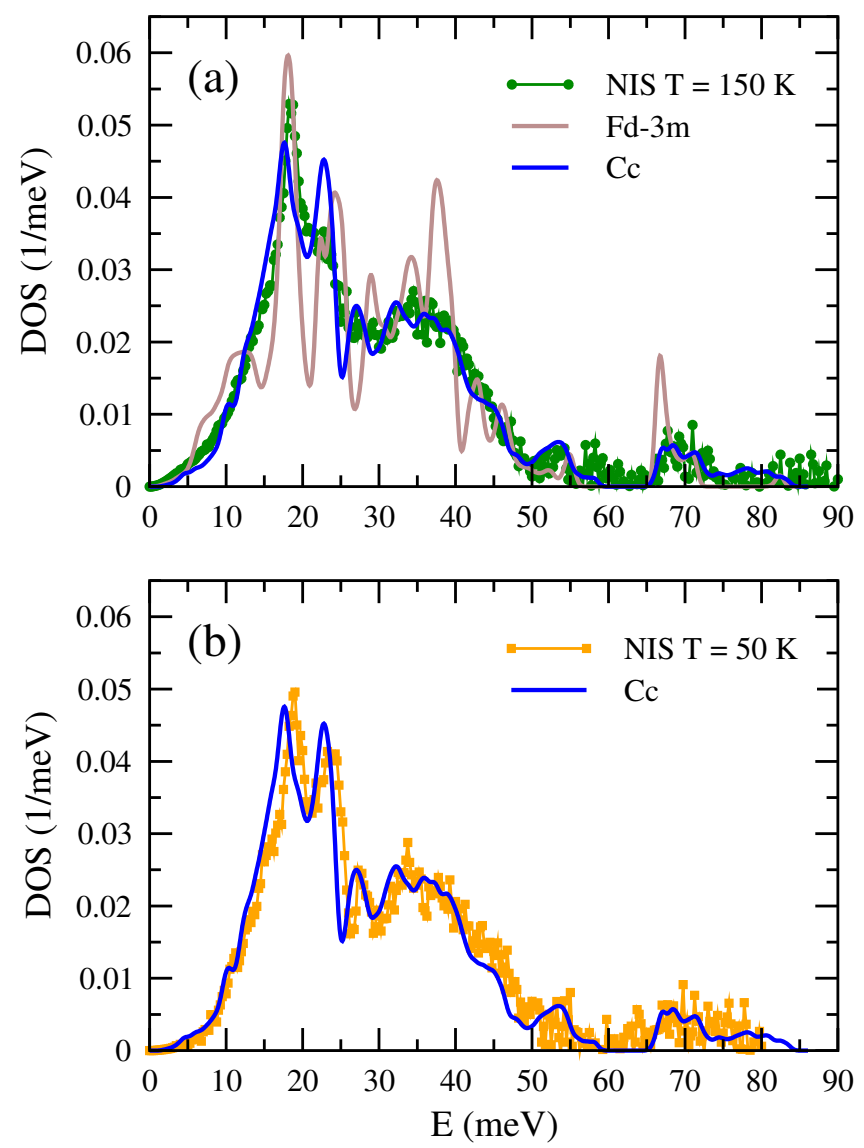

FIG. 6. The Fe-partial phonon DOS in magnetite measured at: (a) $T=150 \mathrm{~K}$ and calculated for the cubic $F d \overline{3} \mathrm{~m}$ and monoclinic $C c$ structure and (b) $T=50 \mathrm{~K}$ and calculated for the monoclinic $C c$ symmetry. The experimental data are taken from Ref. [57].

and around two peaks at $24 \mathrm{meV}$ and $27 \mathrm{meV}$. The main peak at $18 \mathrm{meV}$ is weakly affected by the transition and is only slightly shifted to higher energies below the Verwey transition. The increase of phonon DOS in the cubic phase below $10 \mathrm{meV}$ is related to the quasielastic (diffuse) scattering observed at low energies above the Verwey transition. The stiffening of the monoclinic lattice is evidenced by the increase of the Lamb-Mössbauer factor below $T_{\mathrm{V}}[56]$ and is consistent with the INS measurements [67]. We calculated the first moment of the phonon DOS, which gives the average value of the phonon energy. We found that its value increases, when going from the cubic $(42.09 \mathrm{meV})$ to the monoclinic $(42.22 \mathrm{meV})$ phase. If we take only the contribution from the $\mathrm{Fe}_{B}$ atoms, this change is more pronounced (from $26.74 \mathrm{meV}$ to $27.32 \mathrm{meV}$ ). As we discuss later, the peak at $24 \mathrm{meV}$ is strongly influenced by the $\mathrm{CO}$ order in the $C c$ structure and this effect may explain its modification by the Verwey transition.

The Fe-projected phonon DOS calculated for the cubic and monoclinic symmetries is compared with the experimental data obtained above and below $T_{\mathrm{V}}$ in Fig. 6 .
All theoretical spectra were broadened by a Gaussian with a width of $1 \mathrm{meV}$, which is a typical experimental resolution. In the phonon DOS calculated for the cubic structure, the main peaks coincide with the high intensity DOS measured by NIS. However, apart from the highest peak at $18 \mathrm{meV}$, all other spectral features are much broader than the theoretical peaks. In addition, one finds a broad peak centered at $12 \mathrm{meV}$ not observed in the experimental spectrum. This additional enhancement of phonon DOS results from the anomalous dispersions found in the cubic phase (see Fig. 2).

The phonon DOS calculated for the monoclinic structure shows much better agreement with the experimental data, even for the spectrum obtained above $T_{\mathrm{V}}$. Apart from a small shift of the theoretical energies to lower energies, the positions and widths of all peaks are very well reproduced. The intensity of the lowest peak around 18 $\mathrm{meV}$ is slightly underestimated, while the higher peak around $24 \mathrm{meV}$ is overestimated when compared to the experimental DOS. The position and intensity of the peak around $27 \mathrm{meV}$ agree very well with the experiment.

We emphasize that the theory captures the essential features of the phonon DOS at higher energies which exhibits most of the features obtained by the NIS measurements. Such good agreement demonstrates that the electronic ground state and interatomic forces are very well described by the DFT calculations. It also shows that all atomic positions and their valence states are well defined in the low-temperature monoclinic phase unlike in the high-temperature phase, where the SRO, present in reality, is not modeled by theoretical calculations. We note that the phonon DOS calculated in the $C c$ structure agrees much better with the high-temperature data than the computation in the cubic structure [see Fig. 6(a)]. This difference between the low-temperature and hightemperature structures highlights again the importance of short range correlations above $T_{\mathrm{V}}$.

\section{THE IMPACT OF TRIMERON ORDER ON PHONONS}

In the $C c$ structure, there are 16 nonequivalent $B$ positions. Half of the $\mathrm{Fe}_{B}$ ions have a nominal 2+ valence state and half of them are $3+$. The $\mathrm{CO}$ ordering for each $\mathrm{Fe}_{B}$-O plane (defined here by the fractional coordinate $z$ ) of the primitive $C c$ cell is presented in Fig. 7. The main crystallographic directions $a$ and $b$ of the $C c$ unit cell (see Fig. 1) are rotated by $45^{\circ}$ with respect to the $x$ and $y$ axes defined by the main directions of the cubic phase (both presented at the bottom of the figure). In each plane, the $\mathrm{Fe}_{B}$ atoms form one-dimensional chains with the smallest nearest neighbor distances along the $a$ or $b$ direction. In the $z=0$ and $z=4 / 8$ planes, there are only $\mathrm{Fe}^{2+}$ ions with occupied $d_{x z}$ and $d_{y z}$ orbitals, alternating along the $a$ direction $(B 1, B 2, B 3$, and $B 4$ sites). The $z=1 / 8$ or $z=5 / 8$ planes contain each two 

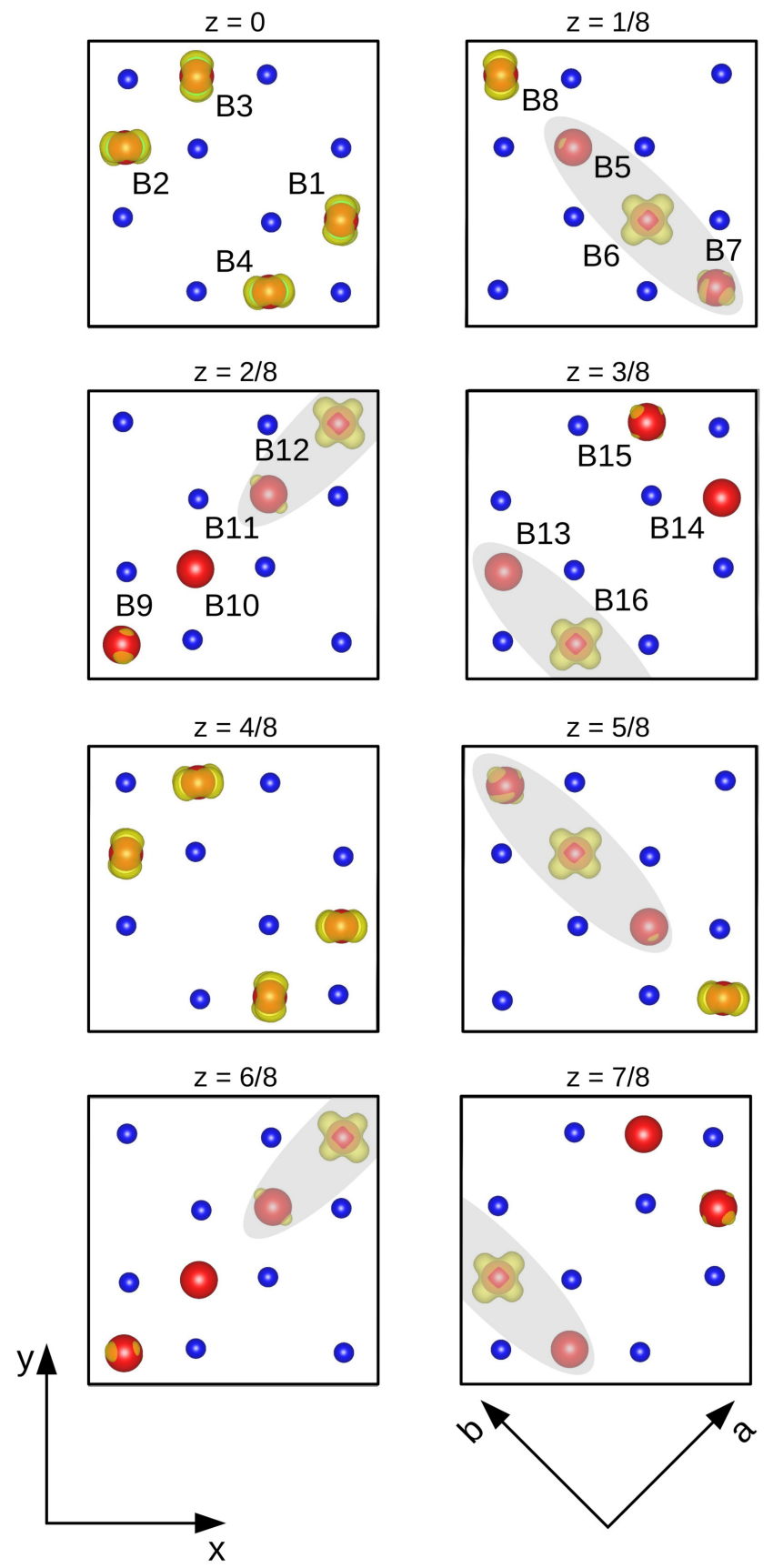

FIG. 7. The charge-orbital order in the $C c$ structure presented for each $(0,0, z)$ plane separately. The trimerons related to the occupied $d_{x y}$ and lying in the $(a, b)$ plane are shown as gray ovals. The $B$ sites are denoted as in Refs. [22, 26].

$\mathrm{Fe}^{2+}$ ions with $d_{x y}(B 6)$ and $d_{y z}$ or $d_{x z}$ orbitals $(B 8)$, while only one $\mathrm{Fe}^{2+}$ ion with the $d_{x y}$ orbital is located in the $z=2 / 8(B 12)$ and $z=3 / 8(B 16)$ planes.

The $\mathrm{Fe}_{B}^{2+}$ ions are the centers of trimerons, which are oriented according to the symmetries of the occupied or-
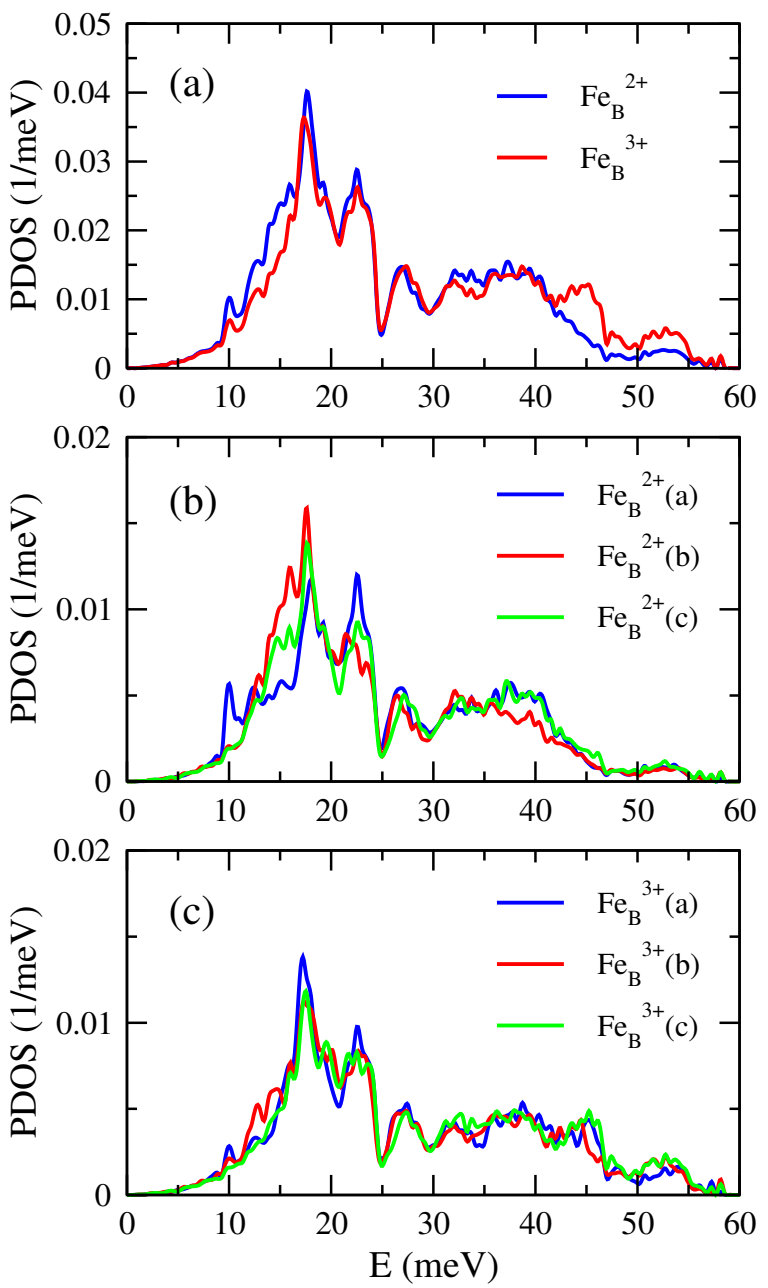

FIG. 8. (a) The partial phonon DOS of $\mathrm{Fe}_{B}^{2+}$ and $\mathrm{Fe}_{B}^{3+}$ ions and the direction-projected phonon DOS of (b) $\mathrm{Fe}_{B}^{2+}$ and (c) $\mathrm{Fe}_{B}^{3+}$ ions in the monoclinic $C c$ structure.

bitals. In Fig. 7, the trimerons with occupied $d_{x y}$ orbitals and oriented along the $a$ or $b$ directions are depicted by the gray ovals. The other trimerons centered at the $B$ sites located in the planes with $z=0,1 / 8,4 / 8$, and $5 / 8$ are aligned along the diagonal $x z$ or $y z$ direction (not shown in Fig. 7). The trimerons are not isolated objects; most of them are connected and have common $\mathrm{Fe}_{B}^{3+}$ ions at their ends. In two cases, the end of one trimeron coincides with the center of the other one located in the $z=0$ or $z=4 / 8$ plane. A detailed analysis of the trimeron order is presented in Refs. [10, 27].

The $\mathrm{CO}$ distribution in the $C c$ structure affects the interatomic forces and therefore also influences the phonon energies. This effect can be clearly observed in Fig. 8(a), where the phonon DOS is plotted for the $\mathrm{Fe}_{B}^{2+}$ and $\mathrm{Fe}_{B}^{3+}$ cations separately. The partial DOS of $\mathrm{Fe}_{B}^{2+}$ ions show higher values at lower energies and the main peaks below $24 \mathrm{meV}$ are larger than for the $\mathrm{Fe}_{B}^{3+}$ ions. In contrast, at higher energies (above $40 \mathrm{meV}$ ), the phonon DOS is dominated by the $\mathrm{Fe}_{B}^{3+}$ vibrations. It can be related to 
an enhanced Coulomb interaction between the Fe cations with the larger valence state and the oxygen anions.

The charge distribution in the orbitals of $\mathrm{Fe}$ ions at the $B$ sites is also highly anisotropic. If we consider two crystallographic directions $a$ and $b$ in the monoclinic $C c$ structure, the differences result from the distribution of charges in the $z=0$ and $z=4 / 8$ planes, in which the chains of $\mathrm{Fe}_{B}^{2+}$ ions are oriented along the $a$ direction, and the $z=1 / 8$ and $z=5 / 8$ planes containing two $\mathrm{Fe}^{2+}$ ions in the chains oriented along the $b$ direction. This anisotropy in charge ordering is related to slightly different values of the lattice parameters along the $a$ and $b$ directions. Therefore, we should also see a variation in the phonon spectra corresponding to these two perpendicular directions.

This expectation is confirmed by the data displayed in Fig. 8(b) and Fig. 8(c), where we present the phonon DOS of $\mathrm{Fe}_{B}^{2+}$ and $\mathrm{Fe}_{B}^{3+}$ vibrations, respectively, along three nonequivalent crystallographic directions of the $C c$ unit cell. Indeed, for these three directions, the phonon DOS exhibits differences which are more significant for the $\mathrm{Fe}_{B}^{2+}$ ions. It shows that the phonon anisotropy results mainly from the orbital ordering of the spin-down states at the $\mathrm{Fe}_{B}^{2+}$ sites. The peak observed at $10 \mathrm{meV}$ is mainly related to the vibrations along the $a$ axis. The phonon DOS above $10 \mathrm{meV}$ and the highest peak around $18 \mathrm{meV}$ show a the anisotropy with the largest contribution from the $b$ component. The peak around $24 \mathrm{meV}$ exhibits even larger anisotropy and is dominated by the vibrations along the $a$ axis.

The influence of the CO anisotropy observed here may explain the significant differences between the NIS spectra measured in the cubic and monoclinic phases around $24 \mathrm{meV}$. One finds that this peak is strongly suppressed in the cubic structure, which can be related to the "melting" of the trimeron order. We expect that the average of the two directions ( $a$ and $b$ ) provides a good model of the dynamical structure factor in the cubic phase. However, this produces too much weight in the $24 \mathrm{meV}$ peak. As less stable trimeron configurations are populated thermally, we expect some phonons to soften. This behavior may explain the transfer of spectral weight from the 24 $\mathrm{meV}$ peak to the $18 \mathrm{meV}$ peak. Irrespective of this feature, we propose that the relative weight of these peaks can be used to measure the degree of SRO.

To better understand the origin of phonon anisotropy between the $a$ and $b$ directions, we analyze the phonon DOS along these two directions for each nonequivalent $\mathrm{Fe}_{B}$ ion. We choose $16 B$ sites belonging to the four lowest planes with $z=0,1 / 8,2 / 8$, and $3 / 8$ (see Fig. 7 ) and present the corresponding phonon DOSs in Fig. 9. In the $z=0$ plane, there are four $\mathrm{Fe}_{B}^{2+}$ ions showing similar DOS. There is a visible anisotropy with the larger contribution of vibrations along the $b$ direction at lower energies. This is caused by the larger Fe-Fe distances and smaller interatomic forces along the direction perpendicular to the $B$-chains. In the $z=1 / 8$ plane, there is some difference between the ions with different valence, with

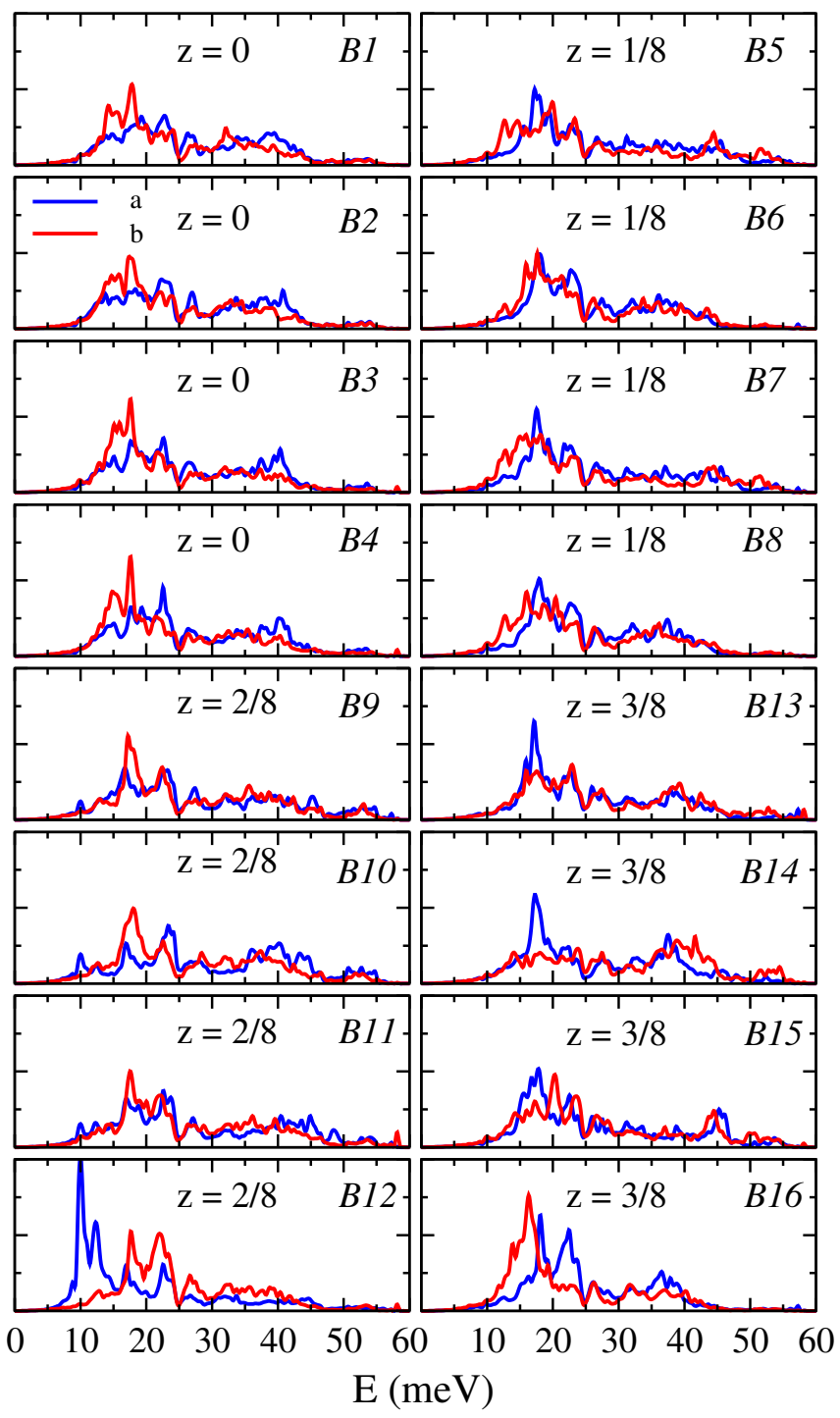

FIG. 9. The phonon DOS projected into all nonequivalent $\mathrm{Fe}_{B}$ ions and two crystallographic directions $a$ and $b$ in the monoclinic structure.

slightly higher anisotropy found for the $\mathrm{Fe}^{3+}$ ions ( $B 5$ and $B 7$ ).

The largest differences are found in the two other planes, each containing only one $\mathrm{Fe}_{B}^{2+}$ ion with an occupied $d_{x y}$ orbital (B12 and $B 16$ ). The DOS at the $B 12$ site along the $a$ direction is strongly shifted to lower energies and exhibits a large peak at $10 \mathrm{meV}$. It shows the greatest shift to lower energies compared to the other $\mathrm{Fe}_{B}$ ions. Interestingly, these vibrations are along the $B$-chains. Similarly, the phonon DOS projected onto the $B 16$ site shows a large anisotropy. Also in this case, the energies of vibrations along the $B$-chains, which in the $z=3 / 8$ plane are parallel to the $b$ direction, are shifted to lower values with a maximum around $15 \mathrm{meV}$. These two peaks at 10 and $15 \mathrm{meV}$ come only from two $B$ sites, and thus their contributions to the total DOS are not very strong. However, they are clearly visible as shoul- 
TABLE I. Mean square displacements of $\mathrm{Fe}_{B}$ ions (in $\AA^{2}$ ) calculated at $T=100 \mathrm{~K}$ and their nominal valence states.

\begin{tabular}{ccccc}
\hline \hline$B$ site & Valence & $\left\langle U_{a}^{2}\right\rangle$ & $\left\langle U_{b}^{2}\right\rangle$ & $\left\langle U_{c}^{2}\right\rangle$ \\
\hline$B 1$ & $2+$ & 0.002307 & 0.002595 & 0.002550 \\
$B 2$ & $2+$ & 0.002323 & 0.002589 & 0.002677 \\
$B 3$ & $2+$ & 0.002301 & 0.002674 & 0.002593 \\
$B 4$ & $2+$ & 0.002266 & 0.002638 & 0.002607 \\
$B 5$ & $3+$ & 0.002271 & 0.002588 & 0.002232 \\
$B 6$ & $2+$ & 0.002257 & 0.002485 & 0.002308 \\
$B 7$ & $3+$ & 0.002229 & 0.002710 & 0.002246 \\
$B 8$ & $2+$ & 0.002272 & 0.002633 & 0.002289 \\
$B 9$ & $3+$ & 0.002305 & 0.002309 & 0.002171 \\
$B 10$ & $3+$ & 0.002231 & 0.002335 & 0.002216 \\
$B 11$ & $3+$ & 0.002343 & 0.002284 & 0.002191 \\
$B 12$ & $2+$ & 0.004078 & 0.002326 & 0.002340 \\
$B 13$ & $3+$ & 0.002340 & 0.002256 & 0.002207 \\
$B 14$ & $3+$ & 0.002298 & 0.002098 & 0.002149 \\
$B 15$ & $3+$ & 0.002299 & 0.002333 & 0.002192 \\
$B 16$ & $2+$ & 0.002293 & 0.002945 & 0.002227 \\
\hline \hline
\end{tabular}

ders in the experimental and theoretical DOSs presented in Fig. 6.

The dynamical anisotropy was further investigated by calculating the mean square displacement (MSD) tensor. The diagonal elements of the MSD tensors of all inequivalent $\mathrm{Fe}_{B}$ atoms obtained for the monoclinic structure at $T=100 \mathrm{~K}$ are presented in Tab. I. The largest value of the MSD is found for the $B 12 \mathrm{Fe}$ atom, which shows the strongest vibrational anisotropy in the phonon DOS. The average displacement of this Fe atom along the $a$ direction exceeds $70 \%$ and is larger than along the $b$ direction. The second largest MSD is for the $B 16$ atom along the $b$ direction. These enhanced atomic displacements should couple strongly to charge fluctuations between $\mathrm{Fe}_{B}$ ions and participate in the polaronic soft modes recently observed in optical experiments [29].

\section{FINAL DISCUSSION AND SUMMARY}

The results presented in the previous section demonstrate a significant impact of the charge-orbital order on lattice dynamics in the monoclinic phase of magnetite. A very good agreement between the calculated and measured phonon spectra indicates that the coupling between the charge-orbital distribution (trimerons) and phonons is well described in our calculations. Through a detailed analysis of the phonon DOS projected into all nonequivalent $B$ sites, we revealed that some trimerons modify strongly the vibrations of central $\mathrm{Fe}_{B}^{2+}$ ions. This trimeron-phonon coupling is especially strong for the trimerons oriented along the main directions of the monoclinic $C c$ structure inducing a large shift of phonon DOS to lower energies. Our results show for the first time that the charge-orbital ordering induce strong anisotropy of the lattice dynamics in the monoclinic phase of magnetite.

The obtained results can be compared with other studies which probe the local charge distribution on $\mathrm{Fe}_{B}$ ions. The NMR and Mössbauer measurements combined with DFT calculations revealed that all $B$-sites can be divided into three groups with the ratio 8:5:3 $[18,19,77,78]$. The first group consists of $8 \mathrm{Fe}^{3+}$ ions, the second group includes $5 \mathrm{Fe}^{2+}$ ions in which electrons occupy the $d_{x z}$ or $d_{y z}$ orbitals, and the third group comprises $3 \mathrm{Fe}^{2+}$ ions with occupied $d_{x y}$ orbitals. The last group of Fe ions is characterized by lower effective magnetic fields and larger electric field gradients [19]. Interestingly, two $B$ sites that show the strongest phonon anisotropy belong to this group of ions (B12 and $B 16$ sites, which correspond to $B 7$ and $B 16$ sites in Refs. $[18,19])$. However, a phonon DOS projected into a single site depends not only on the local valence but also on the charge distribution in the neighborhood of a given atom. This explains the different phonon DOS of the $B 6$ site, which also belongs to the third group, when compared to the $B 12$ and $B 16$ sites.

The present studies demonstrate the influence of static charge-orbital order on phonons. We remark that recent optical conductivity and pump-probe experiments discovered soft electronic modes, which can be explained as the excitations of the trimeron order coupled to atomic vibrations [29]. To model these modes, we considered the coherent tunneling of polarons, which corresponds to charge fluctuations between the $B 12$ and $B 9$ sites in the $z=2 / 8$ plane. Such tunneling induces effectively a shift of the trimeron along the $a$ direction. Similar movement is possible along the $b$ direction in the $z=3 / 8$ plane, where the charge localized at the $B 16$ site can tunnel to the $B 13$ or $B 15$ site. The present calculations show that the trimerons in these two planes couple strongly to the low-energy vibrations with the largest displacements, and therefore they should participate in the polaronic excitations discovered recently by terahertz spectroscopy [29]. These excitations - which propagate along two perpendicular directions - should have different energies due to the anisotropy in lattice and electronic dynamics. This hypothesis can be confirmed in the future by means of polarization-resolved terahertz experiments on untwinned crystals.

We have shown that the existence of the short-range order above the Verwey transition, preserving the local geometry of the monoclinic structure, is well supported by various experiments $[40,41]$. Since the nearest neighbor distances have a dominant impact on interatomic forces and phonon energies, the presence of the short-range order may explain small differences between the phonon spectra measured below and above $T_{\mathbf{V}}$. Moreover, the short-range order explains why the phonon DOS calculated for the $C c$ structure agrees better with the hightemperature NIS data than the computation performed for the perfect cubic structure. Since the deviation from 
the ideal cubic geometry is observed up to the Curie temperature [41], the short-range order may determine the lattice dynamics and the thermodynamic properties of magnetite in a wide range of temperatures.

We suggest that the influence of the static chargeorbital order on phonons found in our studies could still be present above $T_{V}$. However, dynamical effects become very important in the high-temperature phase. The existence of charge fluctuations coupled to phonons is the origin of strong neutron and x-ray diffuse scattering [3438 ] as well as the anharmonic effects revealed by the IXS studies [39]. In this case, both the static displacements from the high-symmetry cubic positions and charge fluctuations may contribute to anharmonic behavior above the Verwey transition.

In summary, we have investigated the lattice dynamical properties of magnetite by performing the DFT calculations for the cubic and monoclinic structures. The results obtained for the low-temperature monoclinic phase show a very good agreement with the experimental data, indicating that the electronic ground state and interatomic forces are well reproduced in the calculations. Most importantly, we have demonstrated that the trimeron order strongly influences the vibrational properties of Fe atoms and this we take as an evidence for strong trimeron-phonon coupling.

In particular, the trimerons with occupied $d_{x y}$ orbitals induce a strong dynamical anisotropy between the main crystallographic directions in the monoclinic structure. This anisotropy is related to the enhanced atomic displacements, which may be closely related to the soft charge modes that were observed in the optical measurements [29]. The phonon density of states calculated for the perfect cubic structure deviates from that of the NIS measurements, which can be explained by the existence of the short-range order in magnetite above the Verwey transition. The local lattice distortions, which modify the charge distribution, influence the interatomic forces and determine the phonon energies at higher temperatures.

\section{Acknowledgements}

This work was supported by the European Regional Development Fund in the IT4Innovations National Supercomputing center-Path to Exascale project, No. CZ.02.1.01/0.0/0.0/16013/0001791 within the Operational Programme Research, Development and Education, and Donau project No. 8X20050 by Ministry and Education of the Czech Republic. P.P. and A.M.O. kindly acknowledge the support by Narodowe Centrum Nauki (NCN, Poland), Projects No. 2017/25/B/ST3/02586 and No. 2016/23/B/ST3/00839. A. M. Oleś is grateful for the Alexander von Humboldt Foundation Fellowship (Humboldt-Forschungspreis). W.T. acknowledges support from the Polish National Agency for Academic Exchange under the "Polish Returns 2019" Program, Grant No. PPN/PPO/2019/1/00014/U/0001. Work at MIT was supported by the US Department of Energy, BES DMSE, Award number DE-FG02-08ER46521 and by the Gordon and Betty Moore Foundation's EPiQS Initiative grant GBMF9459. E.B. and C.A.B. acknowledge additional support from the Swiss National Science Foundation under Fellowships P2ELP2-172290 and P400P2-183842 and the National Science Foundation Graduate Research Fellowship under Grant No. 1122374, respectively.
[1] E. J. W. Verwey, "Electronic conduction of magnetite $\left(\mathrm{Fe}_{3} \mathrm{O}_{4}\right)$ and its transition point at low temperatures," Nature 144, 327 (1939).

[2] E. J. W. Verwey and P. W. Haayman, "Electronic conductivity and transition point of magnetite $\mathrm{Fe}_{3} \mathrm{O}_{4}$," Physica 8, 979 - 987 (1941).

[3] W. H. Bragg, "The structure of magnetite and the spinels," Nature 95, 561 (1915).

[4] E. J. Samuelsen, E. J. Bleeker, L. Dobrzynski, and T. Riste, "Neutron Scattering from Magnetite below 119 K," J. Appl. Phys. 39, 1114 (1968).

[5] T. Yamada, K. Suzuki, and S. Chikazumi, "Electron microscopy of orthorhombic phase in magnetite," Appl. Phys. Lett. 13, 172 (1968).

[6] J. Yoshida and S. Iida, "X-ray diffraction study on the low temperature phase of magnetite," J. Phys. Soc. Jap. 42, 230 (1977).

[7] M. Iizumi, T. F. Koetzle, G. Shirane, S. Chikazumi, M. Matsui, and S. Todo, "Structure of magnetite $\left(\mathrm{Fe}_{3} \mathrm{O}_{4}\right)$ below the Verwey transition temperature," Acta Crystallographica Section B 38, 2121 (1982).
[8] J. P. Wright, J. P. Attfield, and P. G. Radaelli, "Long range charge ordering in magnetite below the Verwey transition," Phys. Rev. Lett. 87, 266401 (2001).

[9] J. P. Wright, J. P. Attfield, and P. G. Radaelli, "Charge ordered structure of magnetite $\mathrm{Fe}_{3} \mathrm{O}_{4}$ below the Verwey transition," Phys. Rev. B 66, 214422 (2002).

[10] M. S. Senn, J. P. Wright, and J. P. Attfield, "Charge order and three-site distortions in the Verwey structure of magnetite," Nature 481, 173 (2012).

[11] D. J. Huang, H.-J. Lin, J. Okamoto, K. S. Chao, H.-T. Jeng, G. Y. Guo, C.-H. Hsu, C.-M. Huang, D. C. Ling, W. B. Wu, C. S. Yang, and C. T. Chen, "Charge-orbital ordering and Verwey transition in magnetite measured by resonant soft x-ray scattering," Phys. Rev. Lett. 96, 096401 (2006).

[12] E. Nazarenko, J. E. Lorenzo, Y. Joly, J. L. Hodeau, D. Mannix, and C. Marin, "Resonant x-ray diffraction studies on the charge ordering in magnetite," Phys. Rev. Lett. 97, 056403 (2006).

[13] J. Schlappa, C. Schüßler-Langeheine, C. F. Chang, H. Ott, A. Tanaka, Z. Hu, M. W. Haverkort, E. Schierle, E. Weschke, G. Kaindl, and L. H. Tjeng, "Direct obser- 
vation of $t_{2 g}$ orbital ordering in magnetite," Phys. Rev. Lett. 100, 026406 (2008).

[14] Y. Joly, J. E. Lorenzo, E. Nazarenko, J.-L. Hodeau, D. Mannix, and C. Marin, "Low-temperature structure of magnetite studied using resonant x-ray scattering," Phys. Rev. B 78, 134110 (2008).

[15] J. E. Lorenzo, C. Mazzoli, N. Jaouen, C. Detlefs, D. Mannix, S. Grenier, Y. Joly, and C. Marin, "Charge and orbital correlations at and above the Verwey phase transition in magnetite," Phys. Rev. Lett. 101, 226401 (2008).

[16] J. Blasco, J. García, and G. Subías, "Structural transformation in magnetite below the Verwey transition," Phys. Rev. B 83, 104105 (2011).

[17] A. Tanaka, C. F. Chang, M. Buchholz, C. Trabant, E. Schierle, J. Schlappa, D. Schmitz, H. Ott, P. Metcalf, L. H. Tjeng, and C. Schüßler-Langeheine, "Symmetry of orbital order in $\mathrm{Fe}_{3} \mathrm{O}_{4}$ studied by $\mathrm{Fe} L_{2,3}$ resonant x-ray diffraction," Phys. Rev. Lett. 108, 227203 (2012).

[18] R. Řezníček, V. Chlan, H. Štěpánková, and P. Novák, "Hyperfine field and electronic structure of magnetite below the Verwey transition," Phys. Rev. B 91, 125134 (2015).

[19] R. Řezníček, V. Chlan, H. Štěpánková, P. Novák, J. Żukrowski, A. Kozłowski, Z. Kakol, Z. Tarnawski, and J. M. Honig, "Understanding the Mössbauer spectrum of magnetite below the Verwey transition: Ab initio calculations, simulation, and experiment," Phys. Rev. B 96, 195124 (2017).

[20] I. Leonov, A. N. Yaresko, V. N. Antonov, M. A. Korotin, and V. I. Anisimov, "Charge and orbital order in $\mathrm{Fe}_{3} \mathrm{O}_{4}$," Phys. Rev. Lett. 93, 146404 (2004).

[21] H.-T. Jeng, G. Y. Guo, and D. J. Huang, "Charge-orbital ordering and Verwey transition in magnetite," Phys. Rev. Lett. 93, 156403 (2004).

[22] H.-T. Jeng, G. Y. Guo, and D. J. Huang, "Chargeorbital ordering in low-temperature structures of magnetite: GGA $+U$ investigations," Phys. Rev. B 74, 195115 (2006).

[23] P. Piekarz, K. Parlinski, and A. M. Oleś, "Mechanism of the Verwey transition in magnetite," Phys. Rev. Lett. 97, 156402 (2006).

[24] P. Piekarz, K. Parlinski, and A. M. Oleś, "Origin of the Verwey transition in magnetite: Group theory, electronic structure, and lattice dynamics study," Phys. Rev. B 76, 165124 (2007).

[25] H. P. Pinto and S. D. Elliott, "Mechanism of the Verwey transition in magnetite: Jahn-Teller distortion and charge ordering patterns," J. Phys.: Condens. Matter 18, 10427 (2006).

[26] K. Yamauchi, T. Fukushima, and S. Picozzi, "Ferroelectricity in multiferroic magnetite $\mathrm{Fe}_{3} \mathrm{O}_{4}$ driven by noncentrosymmetric $\mathrm{Fe}^{2+} / \mathrm{Fe}^{3+}$ charge-ordering: Firstprinciples study," Phys. Rev. B 79, 212404 (2009).

[27] M. S. Senn, I. Loa, J. P. Wright, and J. P. Attfield, "Electronic orders in the Verwey structure of magnetite," Phys. Rev. B 85, 125119 (2012).

[28] The $\mathrm{Fe}^{2+}$ denotes Fe ions with slightly smaller valency and larger occupancy of the minority-spin $t_{2 g}$ orbitals as compared with a $\mathrm{Fe}^{3+}$ ion.

[29] E. Baldini, C. A. Belvin, M. Rodriguez-Vega, I. O. Ozel, D. Legut, A. Kozlowski, A. M. Oleś, K. Parlinski, P. Piekarz, J. Lorenzana, G. A. Fiete, and N. Gedik, "Discovery of the soft electronic modes of the trimeron order in magnetite," Nature Physics 16, 541 (2020).

[30] F. Walz, "The Verwey transition-a topical review," J. Phys.: Condens. Matter 14, R285 - R340 (2002).

[31] T. J. Moran and B. Lüthi, "Elastic and Magnetoelastic Effects in Magnetite," Phys. Rev. 187, 710 (1969).

[32] H. Schwenk, S. Bareiter, C. Hinkel, B. Lüthi, Z. Kakol, A. Kozlowski, and J.M. Honig, "Charge ordering and elastic constants in $\mathrm{Fe}_{3-x} \mathrm{Zn}_{x} \mathrm{O}_{4}$," Eur. Phys. J. B 13, 491 (2000).

[33] K. Chiba, K. Suzuki, and S. Chikazumi, "Diffuse electron scattering from magnetite above the Verwey transition temperature," J. Phys. Soc. Jap. 39, 839 (1975).

[34] Y. Fujii, G. Shirane, and Y. Yamada, "Study of the $123-\mathrm{K}$ phase transition of magnetite by critical neutron scattering," Phys. Rev. B 11, 2036-2041 (1975).

[35] S. M. Shapiro, M. Iizumi, and G. Shirane, "Neutron scattering study of the diffuse critical scattering associated with the Verwey transition in magnetite $\left(\mathrm{Fe}_{3} \mathrm{O}_{4}\right)$," Phys. Rev. B 14, 200 (1976).

[36] Y. Yamada, N. Wakabayashi, and R. M. Nicklow, "Neutron diffuse scattering in magnetite due to molecular polarons," Phys. Rev. B 21, 4642 (1980).

[37] K. Siratori, Y. Ishii, Y. Morii, S. Funahashi, S. Todo, and A. Yanase, "Neutron diffuse scattering study of the high temperature phase of $\mathrm{Fe}_{3} \mathrm{O}_{4}$ - i, determination of atomic displacements at the $\mathrm{x}$ point in the Brillouin zone," J. Phys. Soc. Jap. 67, 2818 (1998).

[38] A. Bosak, D. Chernyshov, M. Hoesch, P. Piekarz, M. Le Tacon, M. Krisch, A. Kozłowski, A. M. Oleś, and K. Parlinski, "Short-range correlations in magnetite above the Verwey temperature," Phys. Rev. X 4, 011040 (2014).

[39] M. Hoesch, P. Piekarz, A. Bosak, M. Le Tacon, M. Krisch, A. Kozłowski, A. M. Oleś, and K. Parlinski, "Anharmonicity due to electron-phonon coupling in magnetite," Phys. Rev. Lett. 110, 207204 (2013).

[40] G. Subías, J. García, and J. Blasco, "EXAFS spectroscopic analysis of the Verwey transition in $\mathrm{Fe}_{3} \mathrm{O}_{4} . "$ Phys. Rev. B 71, 155103 (2005).

[41] G. Perversi, E. Pachoud, J. Cumby, J. M. Hudspeth, J. P. Wright, S. A. J. Kimber, and J. Paul Attfield, "Coemergence of magnetic order and structural fluctuations in magnetite," Nature Communications 10, 2857 (2019).

[42] P. W. Anderson, "Ordering and antiferromagnetism in ferrites," Phys. Rev. 102, 1008 (1956).

[43] D. Ihle and B. Lorenz, "Small-polaron conduction and short-range order in $\mathrm{Fe}_{3} \mathrm{O}_{4}, "$ J. Phys. C: Solid State Physics 19, 5239 (1986).

[44] C. Boekema, R. L. Lichti, K. C. B. Chan, V. A. M. Brabers, A. B. Denison, D. W. Cooke, R. H. Heffner, R. L. Hutson, and M. E. Schillaci, "Experimental evidence for a Mott-Wigner glass phase of magnetite above the Verwey temperature," Phys. Rev. B 33, 210 (1986).

[45] S. K. Park, T. Ishikawa, and Y. Tokura, "Charge-gap formation upon the Verwey transition in $\mathrm{Fe}_{3} \mathrm{O}_{4}$," Phys. Rev. B 58, 3717 (1998).

[46] D. Schrupp, M. Sing, M. Tsunekawa, H. Fujiwara, S. Kasai, A. Sekiyama, S. Suga, T. Muro, V. A. M. Brabers, and R. Claessen, "High-energy photoemission on $\mathrm{Fe}_{3} \mathrm{O}_{4}$ : Small polaron physics and the Verwey transition," Europhys. Lett. 70, 789 (2005).

[47] J. M. Caicedo, J. Fontcuberta, and G. Herranz, "Magnetopolaron-induced optical response in transition metal oxides," Phys. Rev. B 89, 045121 (2014). 
[48] H. Y. Huang, Z. Y. Chen, R.-P. Wang, F. M. F. de Groot, W. B. Wu, J. Okamoto, A. Chainani, A. Singh, Z.-Y. Li, J.-S. Zhou, H.-T. Jeng, G. Y. Guo, Je-Geun Park, L. H. Tjeng, C. T. Chen, and D. J. Huang, "Jahn-Teller distortion driven magnetic polarons in magnetite," Nature Communications 8, 15929 (2017).

[49] Y. Yamada, "Charge ordering and lattice instability in magnetite," AIP Conf. Proc. 24, 79-85 (1975).

[50] M. S. Senn, J. P. Wright, and J. P. Attfield, "The Verwey phase of magnetite - a long-running mystery in magnetism," J. Korean Phys. Soc. 62, 1372 (2013).

[51] M. S. Senn, J. P. Wright, J. Cumby, and J. P. Attfield, "Charge localization in the Verwey structure of magnetite," Phys. Rev. B 92, 024104 (2015).

[52] H. Watanabe and B.N. Brockhouse, "Observation of optical and acoustical magnons in magnetite," Physics Letters 1, 189 (1962).

[53] E. J. Samuelsen and O. Steinsvoll, "Low-Energy Phonons in Magnetite," physica status solidi (b) 61, 615 (1974).

[54] S. Borroni, G. S. Tucker, F. Pennacchio, J. Rajeswari, U. Stuhr, A. Pisoni, J. Lorenzana, H. M. Rønnow, and F. Carbone, "Mapping the lattice dynamical anomaly of the order parameters across the Verwey transition in magnetite," New J. Phys. 19, 103013 (2017).

[55] M. Seto, S. Kitao, Y. Kobayashi, R. Haruki, Y. Yoda, T. Mitsui, and T. Ishikawa, "Site-specific phonon density of states discerned using electronic states," Phys. Rev. Lett. 91, 185505 (2003).

[56] B. Handke, A. Kozłowski, K. Parlinski, J. Przewoznik, T. Ślezak, A. I. Chumakov, L. Niesen, Z. Kakol, and J. Korecki, "Experimental and theoretical studies of vibrational density of states in $\mathrm{Fe}_{3} \mathrm{O}_{4}$ single-crystalline thin films," Phys. Rev. B 71, 144301 (2005).

[57] T. Kołodziej, A. Kozłowski, P. Piekarz, W. Tabiś, Z. Kakol, M. Zajac, Z. Tarnawski, J. M. Honig, A. M. Oleś, and K. Parlinski, "Nuclear inelastic scattering studies of lattice dynamics in magnetite with a firstand second-order Verwey transition," Phys. Rev. B 85, 104301 (2012).

[58] N. W. Grimes, "Off-centre ions in compounds with spinel structure," Phil. Mag.: J. Theor. Exp. Appl. Phys. 26, 1217 (1972).

[59] J. L. Verble, "Temperature-dependent light-scattering studies of the Verwey transition and electronic disorder in magnetite," Phys. Rev. B 9, 5236 (1974).

[60] L. Degiorgi, I. Blatter-Mörke, and P. Wachter, "Magnetite: Phonon modes and the Verwey transition," Phys. Rev. B 35, 5421 (1987).

[61] P. R. Graves, C. Johnston, and J. J. Campaniello, "Raman scattering in spinel structure ferrites," Materials Research Bulletin 23, 1651 (1988).

[62] L. V. Gasparov, D. B. Tanner, D. B. Romero, H. Berger, G. Margaritondo, and L. Forró, "Infrared and Raman studies of the Verwey transition in magnetite," Phys. Rev. B 62, 7939 (2000).

[63] R. Gupta, A. K. Sood, P. Metcalf, and J. M. Honig, "Raman study of stoichiometric and $\mathrm{Zn}$-doped $\mathrm{Fe}_{3} \mathrm{O}_{4}$," Phys. Rev. B 65, 104430 (2002).
[64] M. B. Yazdi, K.-Y. Choi, D. Wulferding, P. Lemmens, and L. Alff, "Raman study of the Verwey transition in magnetite thin films," New J. Phys. 15, 103032 (2013).

[65] A. Kumar, S. Chaudhary, D. K. Pandya, and S. K. Sharma, "Evidence of electron-phonon and spin-phonon couplings at the Verwey transition in $\mathrm{Fe}_{3} \mathrm{O}_{4}$," Phys. Rev. B 90, 024302 (2014).

[66] S. Borroni, J. Teyssier, P. Piekarz, A. B. Kuzmenko, A. M. Oleś, J. Lorenzana, and F. Carbone, "Light scattering from the critical modes of the Verwey transition in magnetite," Phys. Rev. B 98, 184301 (2018).

[67] S. Borroni, E. Baldini, V. M. Katukuri, A. Mann, K. Parlinski, D. Legut, C. Arrell, F. van Mourik, J. Teyssier, A. Kozlowski, P. Piekarz, O. V. Yazyev, A. M. Oleś, J. Lorenzana, and F. Carbone, "Coherent generation of symmetry-forbidden phonons by light-induced electronphonon interactions in magnetite," Phys. Rev. B 96, 104308 (2017).

[68] S. Borroni, G. S. Tucker, U. Stuhr, J. Lorenzana, H. M. Rønnow, and F. Carbone, "Energy domain versus time domain precursor fluctuations above the Verwey transition in magnetite," Phys. Rev. B 101, 054303 (2020).

[69] G. Kresse and J. Furthmüller, "Efficient iterative schemes for ab initio total-energy calculations using a plane-wave basis set," Phys. Rev. B 54, 11169 (1996).

[70] G. Kresse and J. Furthmüller, Comput. Mater. Sci. 6, 15 (1996).

[71] P. E. Blöchl, "Projector augmented-wave method," Phys. Rev. B 50, 17953 (1994).

[72] J. P. Perdew, A. Ruzsinszky, G. I. Csonka, O. A. Vydrov, G. E. Scuseria, L. A. Constantin, X. Zhou, and K. Burke, "Restoring the density-gradient expansion for exchange in solids and surfaces," Phys. Rev. Lett. 100, 136406 (2008).

[73] A. I. Liechtenstein, V. I. Anisimov, and J. Zaanen, "Density-functional theory and strong interactions: Orbital ordering in Mott-Hubbard insulators," Phys. Rev. B 52, R5467 (1995).

[74] K. Parlinski, Z. Q. Li, and Y. Kawazoe, "First-principles determination of the soft mode in cubic $\mathrm{ZrO}_{2}$," Phys. Rev. Lett. 78, 4063 (1997).

[75] K. Parlinski, Software PHONON ver. 6.15, Cracow, Poland (2015).

[76] M. Gajdoš, K. Hummer, G. Kresse, J. Furthmüller, and F. Bechstedt, "Linear optical properties in the projectoraugmented wave methodology," Phys. Rev. B 73, 045112 (2006).

[77] V. Chlan, J. Żukrowski, A. Bosak, Z. Kakol, A. Kozłowski, Z. Tarnawski, R. Řezníček, H. Štěpánková, P. Novák, I. Biało, and J. M. Honig, "Effect of low zn doping on the verwey transition in magnetite single crystals: Mössbauer spectroscopy and x-ray diffraction," Phys. Rev. B 98, 125138 (2018).

[78] T. Kołodziej, I. Biało, W. Tabiś, M. Zubko, J. Żukrowski, K. Łatka, J. E. Lorenzo, C. Mazzoli, Z. Kakol, A. Kozłowski, Z. Tarnawski, E. Wilke, P. Babik, V. Chlan, R. Řezníček, H. Štěpánková, P. Novák, Y. Joly, J. Niewolski, and J. M. Honig, "Magnetic field induced structural changes in magnetite observed by resonant $\mathrm{x}$ ray diffraction and mössbauer spectroscopy," Phys. Rev. B 102, 075126 (2020). 